\title{
Influence of the injection current sweep rate on the polarization switching of vertical-cavity surface-emitting lasers
}

\author{
C. Masoller ${ }^{\text {a) }}$ \\ Departament de Fisica i Enginyeria Nuclear, Universitat Politecnica de Catalunya, Colom 11, \\ E-08222 Terrassa, Spain \\ M. S. Torre \\ Instituto de Física Arroyo Seco, Universidad Nacional del Centro de la Provincia de Buenos Aires, \\ Pinto 399 (7000) Tandil, Argentina \\ Paul Mandel \\ Université Libre de Bruxelles, Optique Nonlinéaire Théorique, Campus Plaine C. P. 231, \\ B-1050 Bruxelles, Belgium
}

(Received 6 June 2005; accepted 1 December 2005; published online 30 January 2006)

\begin{abstract}
We study the polarization switching of vertical-cavity surface-emitting lasers based on simulations of the spin-flip model. We show that the switching point depends on the ramp signal used to scan the injected current. Fast current ramps enlarge the hysteresis region since the switching point moves to high pump values for increasing injection and to low pump values for decreasing injection. The delay of the bifurcation follows a power law with the slope of the current ramp. (C) 2006 American Institute of Physics. [DOI: 10.1063/1.2160711]
\end{abstract}

Vertical-cavity surface-emitting lasers (VCSELs) are emerging as a compact source for high-bit-rate data transmission because they have many advantages over conventional, edge-emitting lasers. A drawback of VCSELs is a polarization instability that arises because the orientation of the polarization of the emitted light is not fixed by geometrical constraints. Due to residual anisotropies (that break the circular transverse symmetry) the output of a VCSEL is linearly polarized along one of two orthogonal directions. When the VCSEL begins to lase one linear polarization dominates, and when the injection current is increased in many devices it is observed that the emission switches to the orthogonal linear polarization. ${ }^{1}$ This polarization switching (PS) phenomenon can be detrimental for polarization-sensitive applications and has received a lot of attention. ${ }^{2-8}$

Usually PS is studied by varying periodically the injected current applied to the VCSEL. The static intensitycurrent response (SI-CR) is usually taken as a reference to predict the response of the device. Studies in nonlinear optics, of the optical transistor, for instance, have shown that this use of the static response is valid only at low modulation frequency of the injected current. ${ }^{9}$ At high modulation frequency, the system cannot follow the injected signal modulation any more and the SI-CR becomes irrelevant. Modulation frequencies are high or low compared to the longest decay rate of the VCSEL. In principle, this is given by the damping rate of the relaxation oscillation frequency (ROF). However, if the device is operated close to a bifurcation point, a critical slowing down is induced which leads to a new and much longer time scale. ${ }^{10}$ In this case, the critical frequency separating high and low frequencies is redshifted.

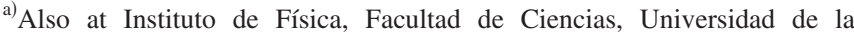
República, Igua 4225, Montevideo 11400, Uruguay; electronic mail: cristina.masoller@upc.edu
}

As a result of critical slowing down, a modulation frequency much lower than the ROF may have to be treated as a fast modulation frequency if the device is operated sufficiently close to a bifurcation. This calls for a careful dynamical analysis of a VCSEL response to a time-varying injected current.

The PS is an example of a parameter swept across a bifurcation. Let us consider a system that has a control parameter $\mu$ and a (static) bifurcation point $\mu_{c}$ such that one state is stable if $\mu<\mu_{c}$ and another state is stable if $\mu>\mu_{c}$. When the control parameter is varied continuously in time from $\mu_{i}<\mu_{c}$ to $\mu_{f}>\mu_{c}$ the bifurcation point is shifted from $\mu_{c}$. In optics a well-known example is the turn-on of a laser, which corresponds to a sweep across a bifurcation representing the transition from the "off" to the "on" state (see Ref. 11 and references therein). It is observed that when a lowfrequency triangular signal is used to scan the injected current (upward and downward) the turn-on and the turn-off are continuous and take place upward and downward at a value that defines the threshold $J=J_{\text {th }}$. This corresponds to a "quasistatic" situation in which the laser reaches the steady state before the current changes appreciably. However, when a high-frequency signal is used the laser turns on suddenly at a value $J^{*}>J_{\text {th }}$, and hysteresis is observed because the laser remains on until $J=J_{\text {th }}$ when the current is decreased.

In this letter we study the PS phenomenon focusing on the influence of the slope of the ramp signal used to scan the injected current. We analyze the polarization-resolved lightcurrent $(L-I)$ characteristic and show that the PS point depends on the slope of the current ramp. For high-frequency ramps, when the current is increased the PS moves to high injection values, and when the current is decreased the PS moves to low injection values. This enlarges the hysteresis region with respect to the quasistatic case of a low-frequency current ramp. We also find a scaling law that relates the delay 
of the bifurcation with the injection current sweep rate.

We use the spin-flip model ${ }^{12}$ that incorporates material birefringence, saturable dispersion, and the spin sublevels of the conduction and valence bands by considering two carrier populations, $N_{+}$and $N_{-}$, with positive and negative spin values. The rate equations for the $x$ - and $y$ - linearly polarized slowly varying complex amplitudes, $E_{x}$ and $E_{y}$, the total carrier density, $N=N_{+}+N_{-}$, and the carrier difference, $n=N_{+}$ $-N_{-}$, are

$$
\begin{aligned}
& \dot{E}_{x, y}= k(1+j \alpha)\left[(N-1) E_{x, y} \pm j n E_{y, x}\right] \mp\left(\gamma_{a}+j \gamma_{p}\right) E_{x, y} \\
&+\sqrt{\beta_{\mathrm{sp}}} \xi_{x, y}, \\
& \dot{N}=\gamma_{N}\left[\mu-N\left(1+\left|E_{x}\right|^{2}+\left|E_{y}\right|^{2}\right)-j n\left(E_{y} E_{x}^{*}-E_{x} E_{y}^{*}\right)\right], \\
& \dot{n}=-\gamma_{s} n-\gamma_{N}\left[n\left(\left|E_{x}\right|^{2}+\left|E_{y}\right|^{2}\right)+j N\left(E_{y} E_{x}^{*}-E_{x} E_{y}^{*}\right)\right] .
\end{aligned}
$$

Here $k$ is the field decay rate, $\gamma_{N}$ is the decay rate of the total carrier population, $\gamma_{s}$ is the spin-flip rate, $\alpha$ the linewidth enhancement factor, and $\gamma_{a}$ and $\gamma_{p}$ are linear anisotropies representing dicroism and birefringence. $\beta_{\mathrm{sp}}$ is the strength of the spontaneous emission noise and $\xi_{x, y}$ are independent Gaussian white noise sources with zero mean and unit variance. $\mu=J / J_{\text {th }}$ where $J$ is the injection current and $J_{\text {th }}$ is the threshold current.

In the absence of anisotropies the model has linearly polarized solutions with arbitrary orientation of the polarization. When $\gamma_{a} \neq 0$ and/or $\gamma_{p} \neq 0$ the solutions are either two orthogonal linear polarizations $\left(\left|E_{x}\right|^{2}=\mu-1,\left|E_{y}\right|^{2}=0\right.$ and $\left.\left|E_{x}\right|^{2}=0,\left|E_{y}\right|^{2}=\mu-1\right)$ or elliptically polarized states. Their stability is determined by the net gain-to-loss ratio, the birefringence, and the saturable dispersion of the material.

We simulated the model equations with typical VCSEL parameters: $k=300 \mathrm{~ns}^{-1}, \alpha=3, \gamma_{n}=1 \mathrm{~ns}^{-1}, \gamma_{s}=50 \mathrm{~ns}^{-1}, \beta_{\mathrm{sp}}$ $=10^{-5} \mathrm{~ns}^{-1}$, and $J_{\mathrm{th}}=1.8 \mathrm{~mA}$. The injection current was increased linearly from $J_{i}=1.6 \mathrm{~mA}$ to $J_{f}=4.5 \mathrm{~mA}$ in a time interval $\Delta T$. To check for bistability and hysteresis, $J$ was then decreased back to $J_{i}$, also in a time interval $\Delta T$. The duration of the current ramp $\Delta T$ was varied from $10^{2}$ ns (fast ramps) to $10^{4} \mathrm{~ns}$ (slow ramps). To simulate the experimental current ramps $J$ was varied in small steps of $d J=0.001 \mathrm{~mA}$.

Figures 1-3 display the $L-I$ curve for different types of PS (for $\gamma_{a}<0$ there is a PS from $x$ - to $y$-, Figs. 1 and 2; for $\gamma_{a}>0$ there is a PS from $y$ - to $x$-, Fig. 3). The left column displays $\left|E_{x}\right|^{2}$ and $\left|E_{y}\right|^{2}$, averaged over a time window of $1 \mathrm{~ns}$ to simulate the bandwidth of the experimental detectors; the right column displays $\left|E_{x}\right|^{2}$ and $\left|E_{y}\right|^{2}$ without averaging, at the laser turn-on. The upper row of Figs. 1-3 corresponds to a fast ramp, the middle row to an intermediate ramp, and the bottom row to a slow ramp.

In all cases there is hysteresis as the PS for increasing current occurs at $\mu=\mu_{1}$, and for decreasing current at $\mu$ $=\mu_{2}<\mu_{1}$. It can be observed that $\mu_{1}$ and $\mu_{2}$ depend on the sweep rate: $\mu_{1}\left(\mu_{2}\right)$ increases (decreases) for fast ramps. The shape of the PS is also modified by the sweep rate: the PS is gradual for fast ramps, while is abrupt for slow ramps. A close inspection of the intensities at the laser turn-on reveals that for fast and intermediate current ramps there is a delay in the turn-on which indicates that the laser did not reach the
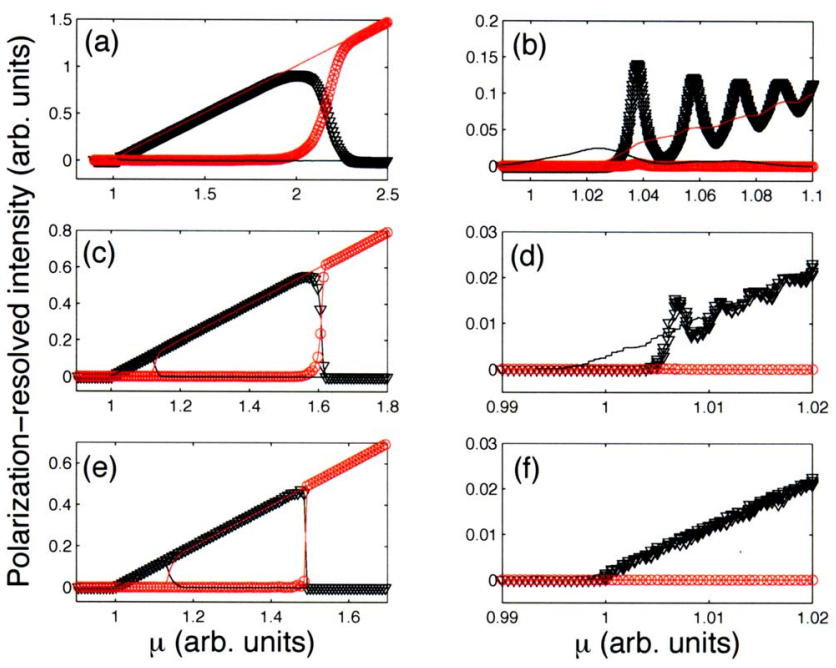

FIG. 1. (Color online) Polarization-resolved $L-I$ curve for $\gamma_{a}=-0.5$ and $\gamma_{p}$ $=0.2 \mathrm{GHz}$. [(a) and (b)] $\left.\Delta T=10^{2} \mathrm{ns;} \mathrm{[(c)} \mathrm{and} \mathrm{(d)}\right] \Delta T=10^{3} \mathrm{~ns} ;[(\mathrm{e})$ and (f)] $\Delta T=10^{4}$ ns. The triangles and circles (red online) represent $\left|E_{x}\right|^{2}$ and $\left|E_{y}\right|^{2}$ for increasing pump. The thin lines represent $\left|E_{x}\right|^{2}$ and $\left|E_{y}\right|^{2}$ (red online) for decreasing pump. The left column shows $\left|E_{x}\right|^{2}$ and $\left|E_{y}\right|^{2}$ averaged in a time window of $1 \mathrm{~ns}$; the right column shows $\left|E_{x}\right|^{2}$ and $\left|E_{y}\right|^{2}$ without averaging at $\mu \sim 1$.

steady state before the current changes; however, in the case of a slow ramp there is no delay: the turn-on occurs at $\mu$ $=1$ indicating that the dynamics corresponds to the static case where the laser reaches the steady state before the current changes appreciably. To support this conclusion we have verified that for slow current ramps the values of $\mu_{1}$ and $\mu_{2}$ agree with those predicted from the linear stability analysis.

In the laser turn-on there is a power-law relationship between the delay in the turn-on and the current sweep rate. ${ }^{10,11}$ To check whether a similar relationship exists in the case of the PS, we did simulations for different $\Delta T$, keeping $\Delta \mu=\mu_{f}-\mu_{i}=\left(J_{f}-J_{i}\right) / J_{\text {th }}$ fixed. Since the PS is a stochastic process, $\mu_{1}$ and $\mu_{2}$ vary with the noise realization. Thus, to study the statistical dependence of $\mu_{1}$ and $\mu_{2}$ with $\Delta T$, for each $\Delta T$ we did several simulations, changing only the noise
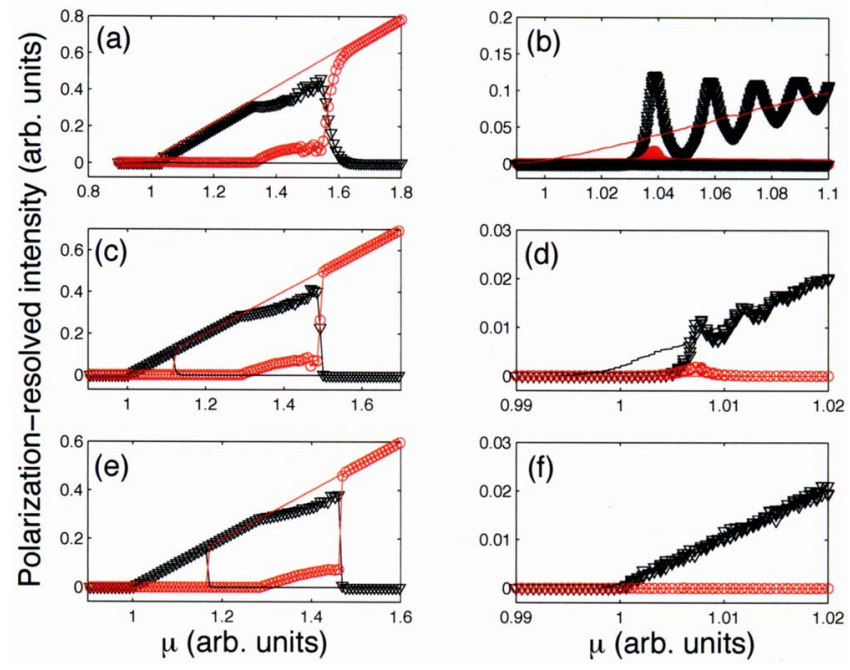

FIG. 2. (Color online) $L-I$ curve for $\gamma_{a}=-0.2$ and $\gamma_{p}=5 \mathrm{GHz}$. [(a) and (b)] $\Delta T=10^{2} \mathrm{~ns} ;[(\mathrm{c})$ and (d) $] \Delta T=10^{3} \mathrm{~ns} ;[$ (e) and (f) $] \Delta T=10^{4} \mathrm{~ns}$. 

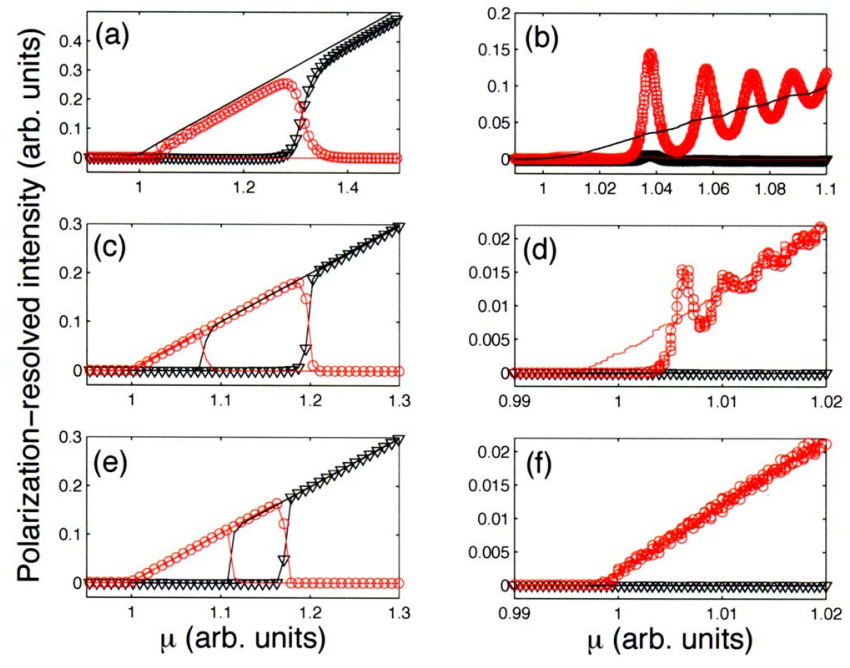

FIG. 3. (Color online) $L-I$ curve for $\gamma_{a}=0.5$ and $\gamma_{p}=50 \mathrm{GHz}$. [(a) and (b)] $\Delta T=10^{2} \mathrm{~ns} ;[(\mathrm{c})$ and (d) $] \Delta T=10^{3} \mathrm{~ns} ;[(\mathrm{e})$ and (f) $] \Delta T=10^{4} \mathrm{~ns}$.

seed. We have also observed that $\mu_{1}$ and $\mu_{2}$ vary with the noise strength, $\beta_{\mathrm{sp}}$, in agreement with the observation of Ref. 6.

The results are displayed in Figs. 4(a) and 4(b), where we plot $\mu_{1}$ and $\mu_{2}$ vs $\Delta T$. We observe that $\mu_{1}\left(\mu_{2}\right)$ decreases (increases) with $\Delta T$. Since the pump varies in small steps, we assume a linear approximation: $\mu(t)=\mu_{i}+b t$ for increasing pump $\left[\mu(t)=\mu_{f}-b t\right.$ for decreasing pump $]$ where $b=\Delta \mu / \Delta T$. Then, the PS occurs at time $t_{1}^{*}=\left(\mu_{1}-\mu_{i}\right) / b$ for increasing
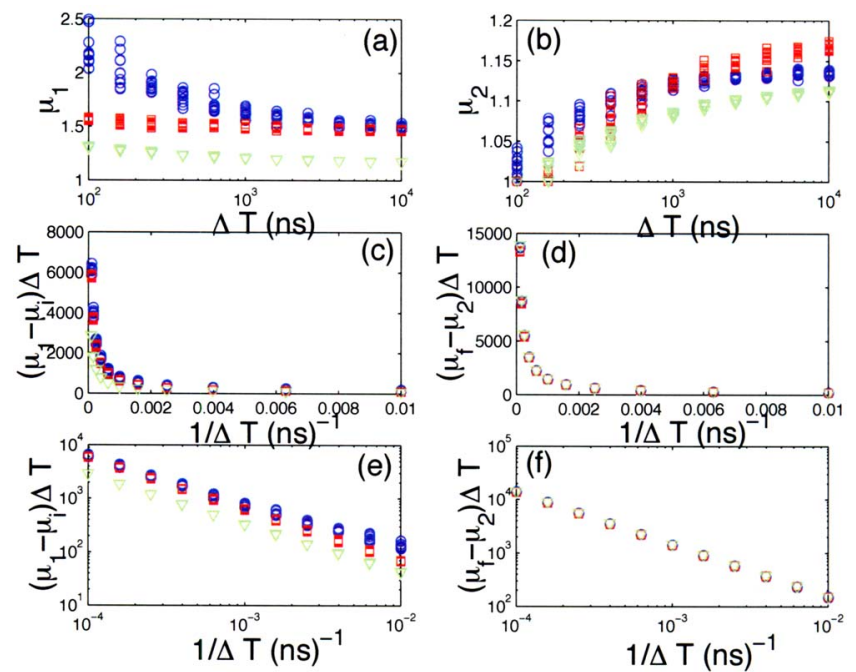

FIG. 4. (Color online) $\mu_{1}$ (a) and $\mu_{2}$ (b) vs $\Delta T$ in a log-linear plot. (c) $\left(\mu_{1}-\mu_{i}\right) \Delta T$ (which is proportional to $t_{1}^{*}$ ) vs $1 / \Delta T$ (which is proportional to the slope of the current ramp). (d) $\left(\mu_{f}-\mu_{2}\right) \Delta T$ (which is proportional to $\left.t_{2}^{*}\right)$ vs $1 / \Delta T$. [(e) and (f)] Log-log plot of (c) and (d). We present results for the parameters of Fig. 1 (circles), Fig. 2 (squares), and Fig. 3 (triangles) and several noise realizations. pump and at $t_{2}^{*}=\left(\mu_{f}-\mu_{2}\right) / b$ for decreasing pump. In Figs. 4(c) and 4(d) we plot $\left(\mu_{1}-\mu_{i}\right) \Delta T \propto t_{1}^{*}$ and $\left(\mu_{f}-\mu_{2}\right) \Delta T \propto t_{2}^{*}$ vs $(\Delta T)^{-1}$. It can be observed that $t_{1}^{*}$ and $t_{2}^{*}$ diverge for slow sweep rates, a phenomenon that reveals "critical slowing down."11 Figures 4(e) and 4(f) display a log-log plot, which shows that a scaling law exists relating $t_{1}^{*}$ and $t_{2}^{*}$ with the slope of the ramp signal.

We have found similar results for other values of the parameters $\gamma_{a}, \gamma_{p}$, and $\gamma_{s}$. Moreover, we have found similar results in simulations of a model for index-guided VCSELs that includes spatial transverse effects. In that model the linear polarizations have different transverse confinements and the carrier equations include diffusion terms that open the opportunity of studying spatial-hole burning effects (the results of the interplay of polarization and spatial effects will be reported elsewhere); however, it has the disadvantage of requiring long simulation times and is not suitable for exploring the power-law relationship displayed in Fig. 4.

To summarize, we have shown that the PS of VCSELs is sensitive to the slope of the signal used to scan the injection current. Fast scans lead to an enlargement of the hysteresis region, compared to the case of a quasistatic, slow current scan. We also found a scaling law relating the bifurcation delay with the current sweep rate. Our results are relevant for polarization-sensitive applications where VCSELs are subject to current modulation.

One of the authors (C.M.) acknowledges support from the "Ramon and Cajal" Program (Spain). Another author (M.S.T.) acknowledges partial support of CONICET Grant No. PIP 2779 and FONCyT Grant No. 3/9598, Argentina. Another author (P.M.) acknowledges partial support of the Fonds National de la Recherche Scientifique (Belgium) and the Interuniversity Attraction Pole Programme-Belgian Science Policy.

${ }^{1}$ K. D. Choquette, D. A. Richie, and R. E. Leibenguth, Appl. Phys. Lett. 64, 2062 (1994)

${ }^{2}$ S. Balle, E. Tolkachova, M. San Miguel, J. R. Tredicce, J. MartinRegalado, and A. Gahl, Opt. Lett. 24, 1121 (1999).

${ }^{3}$ T. Ackemann and M. Sondermann, Appl. Phys. Lett. 78, 3574 (2001).

${ }^{4}$ J. Kaiser, C. Degen, and W. Elsasser, J. Opt. Soc. Am. B 19, 672 (2002).

${ }^{5}$ M. Sciamanna, K. Panajotov, H. Thienpont, I. Veretennicoff, P. Megret, and M. Blondel, Opt. Lett. 28, 1543 (2003).

${ }^{6} \mathrm{M}$. Sondermann, M. Weinkath, and T. Ackemann, IEEE J. Quantum Electron. 40, 97 (2004).

${ }^{7}$ Y. Hong, P. S. Spencer, and K. A. Shore, Opt. Lett. 29, 2151 (2004).

${ }^{8}$ J. Danckaert et al., IEEE J. Sel. Top. Quantum Electron. 10, 911 (2004). ${ }^{9}$ P. Mandel, Opt. Commun. 54, 181 (1985).

${ }^{10} \mathrm{P}$. Mandel, Theoretical Problems in Cavity Nonlinear Optics (Cambridge University Press, Cambridge, England, 1997).

${ }^{11}$ J. R. Tredicce, G. L. Lippi, P. Mandel, B. Charasse, A. Chevalier, and B. Picque, Am. J. Phys. 72, 799 (2004).

${ }^{12}$ J. Martin-Regalado, F. Prati, M. San Miguel, and N. B. Abraham, IEEE J. Quantum Electron. 33, 765 (1997). 